\title{
Innovaciones en ciencias basicas: La transformacion de cursos para el logro de aprendizajes profundos
}

\section{Innovations in basic sciences: The transformation of courses for the achievement of deep learning}

DOI: $10.46932 /$ sfjdv2n5-138

Received in: Oct 1st, 2021

Accepted in: Dec 30th, 2021

\author{
Carmen Soledad Yañez Arriagada \\ Magister en Educación Matemática \\ Universidad Católica de Temuco, Chile \\ Rudecindo Ortega 2950, Temuco, Araucanía \\ E-mail: syanez@uct.cl \\ Valeria Soledad Carrasco Zúñiga \\ Magister en Matemática \\ Universidad Católica de Temuco, Chile \\ Rudecindo Ortega 2950, Temuco, Araucanía \\ E-mail: vcarrasc@uct.cl
}

\section{RESUMEN}

En este trabajo se presenta una experiencia de innovación educativa dirigida a la transformación de la enseñanza y aprendizaje en cursos de ciencias básicas que se imparten en primer año del plan común de dos Facultades de la Universidad Católica de Temuco, Chile. Esta experiencia buscó contribuir al mejoramiento de las prácticas docentes efectivas para el logro de aprendizajes profundos de los estudiantes. Las innovaciones metodológicas que se están implementando en estos curso, corresponden a 'Flipped Classroom' y otras estrategias didácticas como: metodología de proyecto, aprendizaje entre pares, la resolución de problemas contextualizados y Classroom Assessment Tecniques con el apoyo de tecnologías.

Los resultados obtenidos hasta el momento indican un mejoramiento en el rendimiento académico del curso, un rol más activo de los estudiantes lo que se refleja en un mayor involucramiento e interes por parte de ellos, al igual que un cambio en el modelo docente de uno centrado en la enseñanza a uno con énfasis en el aprendizaje de los estudiantes. Para ello, se realizó un rediseño del curso basado en el 'alineamiento constructivo' entre los resultados de aprendizaje, su forma de evaluación y sus actividades de aprendizaje.

Palabras clave: Transformación de cursos, Flipped Classroom, Aprendizajes Profundos, Ciencias Básicas.

\footnotetext{
ABSTRACT

This paper presents an experience of educational innovation aimed at transforming teaching and learning in basic science courses taught in the first year of the common plan of two faculties of the Catholic University of Temuco, Chile. This experience sought to contribute to the improvement of effective teaching practices for the achievement of deep learning of students. The methodological innovations that are being implemented in these courses correspond to 'Flipped Classroom' and other didactic strategies
} 
such as: project methodology, peer learning, contextualized problem solving and Classroom Assessment Techniques with the support of technologies.

The results obtained so far indicate an improvement in the academic performance of the course, a more active role of the students which is reflected in a greater involvement and interest on their part, as well as a change in the teaching model from one focused on teaching to one with emphasis on student learning. To this end, a course redesign was carried out based on the 'constructive alignment' between learning outcomes, assessment and learning activities.

Keywords: Course transformation, Flipped Classroom, Deep Learning, Basic Sciences.

\section{INTRODUCCIÓN}

La UC Temuco respondiendo a la mejora del proceso de enseñanza y aprendizaje, implementa un Modelo educativo por Competencias, centrado en el estudiante. Puesto que la formación por competencias, pretende que los estudiantes se formen de forma eficiente en su contexto; generando aprendizajes significativos cuyos conocimientos preparará al alumno para su futura vida laboral (Valdez, Flores, Cortés ,2021). Esto ha llevado una renovación curricular. El problema actual se focaliza en las dificultades asociadas al aprendizaje de los estudiantes en los cursos de Ciencias Básicas de primer año de la UC Temuco, específicamente en la asignatura de Álgebra y Cálculo. Estos cursos presentan, altas tasas de reprobación, estudiantes pasivos, metodologías de enseñanza tradicionales y mala utilización de recursos tecnológicos existentes. Respondiendo a estas dificultades, el año 2013 se genera un proceso de transformación de cursos de ciencias básicas para el logro de aprendizajes profundos, introduciendo para ellos diversas innovaciones metodológicas y recursos educativos tecnológicos de última generación.

\section{DESARROLLO}

\subsection{MARCO TEÓRICO}

El sistema de la educación superior en Chile ha sufrido profundas transformaciones en las últimas décadas, de un sistema altamente selectivo y homogeneo se ha pasado a uno caracterizado por la masificación y heterogeneidad de la matrícula (Lemaitre, 2005; De los Ríos 2009).

Este fenómeno ha significado la presencia de nuevos estudiantes. Si hace un par de décadas atrás las aulas universitarias estaban pobladas mayoritariamente de estudiantes 'academicos' caracterizados por una orientación profunda hacia el aprendizaje y minoritariamente de estudiantes 'no académicos, que presentaban una orientación superficial hacia un aprendizaje. Hoy en día, esta relación se ha invertido. (Biggs, 2006)

La incorporación masiva de estos nuevos estudiantes, ha generado un profundo cuestionamiento en las formas en que tradicionalmente se ha ejercido la docencia universitaria, demandando un cambio de 
modelo, de uno centrado en la enseñanza del profesor a otro centrado en el aprendizajes de los estudiantes (Biggs, 2006).

Apartir de esta realidad, es que se opta por iniciar un proceso de transformación de la enseñanza y aprendizaje en cursos de Ciencias Básicas de primer año de la Universidad Católica de Temuco, impulsando algunas innovaciones metodológicas que constribuyan a este propósito.

El proceso de transformación de cursos se sustenta en algunas premisas: 1) Las decisiones y acciones que se realicen en un curso deben tributar hacia el aprendizaje de todos los estudiantes. 2) Una docencia es eficaz en la medida que permita el logro de aprendizajes profundos en los estudiantes. 3) Los estudiantes tienen una orientación profunda hacia el aprendizaje cuando se les provee experiencias de enseñanza y aprendizaje activas, significativas y contextualizadas. 4) Los resultados de aprendizaje que se espera que los estudiantes demuestren al finalizar un curso deben formularse en términos de desempeños o actuaciones complejas que se evidencian en situación. 5) El diseño de un curso transformado debe sustentarse en el principio de 'alineamiento constructivo'. (Biggs, 2006; Dee Fink, 2013)

La transformación de los cursos de ciencias básicas, ha sido desarrollada en diferentes instituciones de educación superior especialmente en Estados Unidos y Canada. Esto ha permitido que los cursos se alineen con las nuevas tendencias y modelos educativos en educación superior, requerimientos institucionales y lineamientos de las políticas públicas. Uno de los beneficios de la transformación de cursos es que promueve el involucramiento de los estudiantes con impacto en la calidad de sus aprendizajes (University of Texas, 2012). Estas transformaciones se han llevado a cabo en cursos como física, biología, matemáticas, química, y en todos estos casos la transformación ha implicado la incorporación, en distinta medida, de tecnologías educativas. Las Universidades que han iniciado este proceso reportan mejoras significativas en el aprendizaje, y por ende en el rendimiento académico de los estudiantes.

Las innovaciones metodológicas impulsadas en el marco de la transformaciónn de cursos, corresponde a: 'Flipped Classroom', Método de Proyecto, Aprendizaje entre Pares, Contextualización de las Matemáticas y Classroom Assessment Tecniques (CATs).

a) La 'Flipped Classroom' (o aula invertida). Es un modelo centrado en el estudiante que deliberadamente consiste en trasladar una parte o la mayoría de la clase 'expositiva - tradicional' (Centrada en la transmisión de información o entrega de conceptos) al exterior del aula, para aprovechar el tiempo en clase maximizando las interacciones entre profesor y estudiante y entre pares para lograr niveles de mayor profundidad en el aprendizaje. La idea es que las actividades de baja complejidad sea realizadas por los estudiantes de forma autónoma antes de la clase presencial, para que en ésta, se desarrollen actividad que impliquen mayor complejidad y sean abordadas colaborativamente entre los 
estudiantes, con la facilitación activa del docente (Observatorio de Innovaciones Educativas TEC, 2014).

b) El Método de Proyecto. Corresponde a una metodología donde los estudiantes trabajan de manera activa, planean, implementan y evalúan proyectos a partir de la resolución de problemas prácticos que tienen aplicación en el mundo real más allá del aula de clase (Blank, 1997; Harwell, 1997; Martí, 2010).

c) Aprendizaje entre Pares. Es una técnica interactiva propuesta por el profesor Eric Mazur de la Universidad de Harvard, que promueve la discusión en pequeños grupos de estudiantes, favoreciendo de este modo el aprendizaje entre pares y la discusión entre sujetos que presentan similar congruencia cognitiva. Si bien estas discusiones deben ser supervisadas por el o los docentes a cargo del curso, permiten a los estudiantes compartir sus dificultades y experiencias personales en la construcción activa de aprendizajes. Para que este método sea realmente efectivo los estudiantes deben concurrir al aula con información base necesaria para abordar el trabajo en clases (Lasry, Mazur y Watkins, 2008).

d) Contextualización de la Matemática. Este modelo plantea la vinculación entre las matemáticas y las ciencias que la requieren, así como la vinculación con situaciones profesionales y de la vida cotidiana (Camarena 2008). Contribuye en la adquisición de las competencias matemáticas tales como: a) pensar matemáticamente; b) plantear y resolver problemas matemáticos; c) modelar matemáticamente; d) argumentar matemáticamente; e) representar entidades matemáticas (situaciones y objetos); f) utilizar los símbolos matemáticos; g) comunicarse con las matemáticas y comunicar sobre matemáticas h) utilizar ayudas y herramientas (incluyendo las nuevas tecnologías) (Camarena, 2013).

e) Classroom Assessment Tecniques (CATs). Corresponde a técnicas de seguimiento de los aprendizajes en clase, cuyo propósito es mejorar la calidad del aprendizaje de los estudiantes durante el proceso de enseñanza y aprendizaje, proporcionando al docente comentarios acerca de la eficacia de sus prácticas docentes a través del progreso de los estudiantes (Angelo y Cross,1998 citando en Enerson, Plank, Johnson 2007).

\subsection{DESCRIPCIÓN DE LA INNOVACIÓN}

El primer semestre 2015, en los cursos Cálculo I de la Facultad de Ingeniería y Algebra de la Facultad de Recursos Naturales, se inicia su transformación con la aplicación de un diagnóstico situacional que permita la reformulación de los Resultados y rediseño de la Guía de Aprendizaje, sobre la base del 'alineamiento constructivo' (Biggs, 2006)

El rediseño de los cursos se orienta hacia un aprendizaje significativo y profundo, centrado en el alumno, que logre fortalecer su autonomía. Para ello se utilizó como eje central el método de enseñanza y aprendizaje, la 'Flipped clasroom' con apoyo tecnológico a través de la plataforma Moodle en la UCT (Leraning Management System) y con uso de tecnologías de última generación(videos, cuestionarios 
on-line, Google Drive, LMS), además de otras estratégias didácticas complementarias, como: Aprendizaje entre pares; Metodología de proyecto; Resolución de problemas contextualizados , como una estrategia que facilita la modelización de situaciones cotidianas y de su futura profesión y CAT'S como estrategia de seguimiento y monitoreo que permite dar cuenta del nivel de avance en el logro de los resultados de aprendizaje. Y con una retroalimentación justo a tiempo.

\subsection{PROCESO DE IMPLEMENTACIÓN DE LA INNOVACIÓN}

La implementación de esta experiencia se llevo a cabo durante el desarrollo semestral de los cursos.

En una primera fase se seleccionaron y capacitaron ayudantes de aprendizaje, con el fin de apoyar la implementación de la 'Flipped Classroom'. Mediante el uso de herramientas en linea los estudiantes accedieron a los contenidos del curso con un nivel de complejidad inicial, a través del uso de videos que son utilizados por los estudiantes durante sus horas autónomas, dichos videos son preparados por los profesores y grabados por docentes y ayudantes, con una duración no superior a 10 minutos, para luego ser publicados en la plataforma. Para constatar la revisión de éstos los estudiantes responden cuestionarios on-line, y/o redactan resúmenes de sus aprendizaje surgidos a partir de cada video.

Posteriormente, los estudiantes en el aula realizan discusiones, debates, consultas con sus pares. El docente fomenta su participación activa, lo que lleva a un aprendizaje colaborativo y entre pares, que evidencia que en estas metodologías el docente pasa a ser un facilitador del aprendizaje fomentando el protagonismo del estudiante. En definitiva, los estudiantes desarrollan actividades de trabajo de mayor complejidad en forma colaborativa en clases teniendo como base los contenido tratado en cada video.

Las evaluaciones realizadas en los cursos, corresponden a dos tipos: 1) evaluación focalizada de contenidos (de proceso o parciales) consistente en pruebas, controles, talleres; 2) Evaluación de desempeño, que implica evaluar en situación los resultados de aprendizaje definidos para el curso, los cuales a su vez integran niveles de competencias específicas (aquellas propias de la carrera) y genéricas (como aprendizaje autónomo y trabajo en equipo). Se utilizó la Metodología de Proyecto a través de la cual los estudiantes desarrollaron un proyecto que buscó integrar la teoría y el desempeño profesionalizante en cada uno de los resultados de aprendizaje establecidos para el curso. Además, se implementó la contextualización de la matemática en los proyectos asignados, como también en las guías de trabajo y apuntes del curso de forma transversal durante todo el semestre. Así también, se utilizaron Classroom Assessment Tecniques (CATs), como una herramienta eficiente para el seguimiento de los aprendizajes en clase, entre las que se llevaron a cabo el punto fangoso y papel en un minuto. 


\subsection{EVALUACIÓN DE RESULTADOS}

Los resultados que podemos señalar a partir de los procesos de transformación de la enseñanza y aprendizaje en estos cursos, han permitido identificar fortalezas y debilidades. A nivel de aula, las fortalezas observadas fueron: mejora significativa del ambiente de trabajo, transformación de la clase en un espacio de interactividad, incentiva la resolución de problemas en aula, mayor y mejor comunicación entre docente y estudiante.

Para el estudiante se potencia el trabajo autónomo y colaborativo, el hábito de estudio, mejora su organización y distribución del tiempo; comienza a ser el constructor de su propio aprendizaje, optimiza y aprovecha los recursos entregados, hace mejor y mayor uso de los recursos para un aprendizaje profundo.

Por otro lado, el docente pasa a tener un rol de facilitador y guía con el estudiante, toma como eje central del proceso de enseñanza y aprendizaje, el aprendizaje profundo y significativo del estudiante, centra su retroalimentación en dificultades individuales más que grupales apoyando a cada individuo según sus necesidades, comienza a abandonar la clase puramente expositiva y a replantearse su papel en la sala de clases, es decir, pasa a tener una mayor reflexión y autocrítica de su docencia.

También es posible dar a conocer algunas debilidades identificadas en la implementación, específicamente en la Flipped Classroom o clase al revés, y que se refiere al cambio de roles que se requieren para desempeñarse con éxito en estos cursos. Los estudiantes generalmente llegan a la universidad asumiento un rol pasivo como repectores de contenidos, y les resulta dificil el cambio a un rol activo y de responsables de la construcción de sus aprendizajes, este cambio no se da instantáneamente, se requiere motivar, formar, concientizar al estudiante para que asuma este nuevo rol. Por su parte, los docentes acostumbrados a realizar clases expositivas también necesitan preparación para asumir el desafío que implica estas nuevas metodologías.

\section{CONCLUSIONES}

Teniendo en cuenta que esta innovación aún está en proceso de pilotaje, igualmente es posible establecer algunas conclusiones:

- La Metodología Flipped Classroom ha sido positiva para alumnos con un tipo de aprendizaje concreto, ya que facilita que este tipo de estudiante transite 'naturalmente' hacia niveles de mayor abstracción, desarrollando actividades que van de una menor a mayor complejidad cognitiva.

- Los estudiantes valoran positívamente la cantidad y calidad de recursos disponibles en plataforma.

- Se fomenta y profundiza entre los estudiantes y docentes el trabajo colaborativo entre pares.

- El rol del estudiante ha cambiado desde un rol pasivo a uno activo y crítico.

- El estudiante ha sido capaz de guiar su propio aprendizaje, mejorando su autonomía. 
- La incorporación de tecnologías de última generación motiva al alumno al seguimiento y cumplimiento de las actividades de enseñanza -aprendizaje.

- La incorporación de tecnologías de última generación para los docentes ha sido una instancia para explorar las posibilidades que nos ofrecen estos recursos digitales para hacer tareas que serían imposible o de una mayor dificultad sin ellos.

- Aumento de un clima positivo en la sala de clases, lo que invita al aprendizaje profundo y significativo. 


\section{REFERENCIAS}

Angelo y Cross 1993 citado en Enerson, D., Plank, K., \& Johnson, N. (2007). An Introduction to Classroom Assessment Techniques. In Classroom Assessment Techniques . Retrieved June 19, 2015.

Biggs, J. (2006). Calidad del aprendizaje universitario (2da ed.). Madrid: Narcea.

Camarena, P., (2008). Teoria de la Matemática en el Contexto de las Ciencias. Actas del III Coloquio Internacional sobre Enseñanza de las Matemáticas, Conferencia Magistral. Lima, Perú.

Dee Fink, L. (2013). Creating significant learning experiences: An integrated approach to designing college courses. (2da ed.). San Francisco, California: Jossey-Bass.

De los Rios, D. (2009). Retención de estudiantes vulnerables en la educación Universitaria Chilena. Calidad En La Educación, 50-83.

Lasry, N., Mazur, E., \& Watkins, J. (2008). Peer instruction: From Harvard to the two-year college. American Journal of Physics, 76, 1066-1066. doi:10.1119/1.2978182

Lemaitre, M. (2005). La Calidad Colonizada: Universidad y globalización. Revista De La Educación Superior, 34(1), 123-134.

Martí, J., Heydrich, M., Rojas, M., \& Hernández, A. (2010). Aprendizaje basado en proyectos: Una experiencia de innovación docente. Revista Universidad EAFIT, 46(158), 11-21.

Observatorio de Innovación Educativa del Tecnológico de Monterrey. (2014, October). Aprendizaje Invertido . In Reportes EduTrends. Retrieved June 19, 2015.

Trejo, E., Camarena, P., \& Trejo, N. (2013). Las matemáticas en la formación de un ingeniero: La matemática en contexto como propuesta metodológica. Revista De Docencia Universitaria, 11, 397-424.

University of Texas at Austin. (2012). Transforming courses, learning and success . In What starts here changes de world. Retrieved June 19, 2015.

Valdez, E. Flores, M. \& Cortés,G. , (2021). Preparando a las nuevas generaciones con un enfoque organizacional tipo Hollywood a través del aprendizaje basado en Proyectos colaborativos. Revista South Florida Journal of Development, Miami, v. 2, n. 5, oct./dec. 2021 /.DOI:

https://doi.org/10.46932/sfjdv2n5-029 